\title{
ADDENDA TO "A VARIATIONAL PROBLEM RELATED TO AN OPTIMAL FILTER PROBLEM WITH SELF-CORRELATED NOISE"
}

\author{
BY \\ LEONARD D. BERKOVITZ( $\left.{ }^{(}\right)$AND HARRY POLLARD
}

\begin{abstract}
The explicit solution is given of a nonclassical variational problem that is related to an optimal filter problem.
\end{abstract}

1. Introduction. In [1] we showed that the following variational problem has a unique solution and we gave a partial characterization of the solution. We also discussed the relationship of the variational problem to an optimal filter problem.

Problem. Let $Y$ denote the class of functions $y$ that are in $L_{1}[0, \infty]$, that are absolutely continuous, that satisfy

$$
y(0)=0,
$$

and have the property that the function $G$ defined by

$$
G(u)=-y^{\prime}(u)+e^{-u}+e^{-u} \int_{0}^{u} e^{t} y^{\prime}(t) d t
$$

is in $L_{2}[0, \infty]$. Minimize the functional

$$
J(y)=\left(\int_{0}^{\infty}|y| d t\right)^{2}+\int_{0}^{\infty} G^{2} d t
$$

in the class $Y$.

In this paper we shall give the explicit solution of the variational problem. As in [1] we denote the unique minimizing function by $z$ and we denote by $F$ the function obtained by taking $y^{\prime}=z^{\prime}$ in the right-hand side of (1.2). We denote the value of the minimum of the functional $J$ by $m$ and let

$$
I=\int_{0}^{\infty}|z| d t
$$

In [1] we showed that $z$ has compact support $(0, \lambda)$ and that on its support $z$ is composed of a finite number of contiguous quartic arches whose signs alternate. In this paper we show that on its support $z$ consists of precisely one quartic arch. The principal result of this paper is the following theorem.

Received by the editors April 10, 1970.

AMS 1969 subject classifications. Primary 4900, 9340; Secondary 9410.

Key words and phrases. Nonclassical variational problem, optimal min-max filter.

(1) The work of this author was supported by NSF Grant GP-11477.

Copyright (C) 1971, American Mathematical Society 
THEOREM 3. The minimizing function is

$$
\begin{aligned}
z(u) & =q(u), & & 0 \leqq u \leqq \lambda, \\
& =0, & & u \geqq \lambda,
\end{aligned}
$$

where

$$
q(u)=-\frac{I u^{4}}{24}+\frac{(1-v)^{2} u^{3}}{12}+\frac{\left(2 I+v^{2}-1\right) u^{2}}{4}+\nu u
$$

and $\nu=(1-2 m)^{1 / 2}$. The numerical values of the constants, correct to six decimal places, are

$$
\begin{aligned}
m & =0.478318, & I & =0.106953, \\
\nu & =0.208234, & \lambda & =2.732889 .
\end{aligned}
$$

Note that since $z$ and $z^{\prime}$ are continuous (the absolute continuity of $z^{\prime}$ is proved in Theorem 2 of [1]), the quartic $q$ has a double zero at $u=\lambda$.

The remainder of this paper is a continuation of [1]. We assume that the reader has [1] at hand. We use the notation and numbering of [1]. Thus, the next section of this paper will be numbered 10 since the last section of [1] is numbered 9 .

10. Further characterization of $z$.

LEMma 11. Let I be given by (5.3), let $m$ be the value of the minimum, and let

$$
\nu=(1-2 m)^{1 / 2} \text {. }
$$

Then

$$
\begin{aligned}
& z^{\prime}(0)=(1-2 m)^{1 / 2}=\nu \\
& z^{\prime \prime}(0)=I-m=\left(2 I+\nu^{2}-1\right) / 2 \\
& z^{m}(0)=1-m-(1-2 m)^{1 / 2}=(1-\nu)^{2} / 2 .
\end{aligned}
$$

Since $0<m<1 / 2$ (Lemma 7), the quantity $\nu$ defined in (10.1) is real. Equation (10.2) is an immediate consequence of (9.1), (9.2), and (10.1). Equation (10.3) follows from Corollary 2 of Lemma 9 and from (10.1). To establish (10.4) we proceed as follows. From (8.10) and (8.7) we get

$$
z^{m}(u)=-\Psi^{\prime}(u)=F(u)-\Psi(u) .
$$

If we set $u=0$ in the preceding equation and use (8.1), (5.8) and (5.7), we get

$$
z^{m}(0)=F(0)-m=F^{2}(0) / 2 .
$$

If we now use (9.2) and (10.2) we obtain the first equality in (10.4). The second follows from (10.1).

Another useful relationship is

$$
1-2 m=\int_{0}^{\lambda} e^{-t} z^{\prime}(t) d t,
$$


which is established as follows. Substitute (1.2), with $\dot{y}^{\prime}$ replaced by $z^{\prime}$ and $G$ replaced by $F$, into (5.8) and interchange the order of integration in the iterated integral.

As a corollary of the next lemma we shall obtain an inequality involving $I$ and $\nu$ that will be used to show that $z$ has only one arch.

LEMMA 12. Let

$$
K=2 \int_{0}^{\infty} e^{-u} g^{\prime}(u) d u+\int_{0}^{\infty}(1+u) e^{-u} z(u) d u,
$$

where $g$ is defined by (6.7). Then

$$
K=\left(9 v^{2}-4 v+1\right) / 2 .
$$

In the course of showing that $F$ is absolutely continuous we showed that $g$ is absolutely continuous and that $\left|g^{\prime}(u)\right| \leqq I[1$, p. 166, first sentence]. Hence the first integral in (10.7) is finite. Moreover, from the inequality $\left|g^{\prime}(u)\right| \leqq I$ and the inequality $(1+u) \exp (-u) \leqq 1$ for $u \geqq 0$, we obtain the following consequence of Lemma 12:

$$
\left[9 v^{2}-4 v+1\right] / 6 \leqq I .
$$

We now proceed to establish Lemma 12. From (7.8), (6.7), and (8.5), we get that

$$
g^{\prime}(u)=z^{\prime \prime}(u)+e^{u} \int_{u}^{\infty} e^{-t} F(t) d t
$$

If we substitute this expression for $g^{\prime}$ into the right-hand side of (10.7), we get

$$
K=2 \int_{0}^{\infty} e^{-u} z^{\prime \prime}(u) d u+2 \int_{0}^{\infty} d u \int_{u}^{\infty} e^{-t} F(t) d t-\int_{0}^{\infty} z(u) d\left((u+2) e^{-u}\right) .
$$

Since $z^{\prime}$ is absolutely continuous (Theorem 2 of [1]) we may write $z^{\prime \prime}(u)$ as $d z^{\prime}(u)$ in the first integral in (10.9). We now develop the right-hand side of (10.9) as follows. Interchange the order of integration in the second integral and perform the resulting integration with respect to $t$. In the first and third integrals, integrate by parts, taking into account $z(0)=z(\infty)=z^{\prime}(\infty)=0$ and (10.2). The result is

$$
K=-2 \nu+4 \int_{0}^{\infty} e^{-u} z^{\prime}(u) d u+2 \int_{0}^{\infty} t e^{-t} F(t) d t+\int_{0}^{\infty} u e^{-u} z^{\prime}(u) d u .
$$

If we now use (10.6), we get

$$
K=4-2 \nu-8 m+\int_{0}^{\infty} u e^{-u}\left(2 F(u)+z^{\prime}(u)\right) d u .
$$

Let $L$ denote the integral on the right-hand side of (10.10). If we use (1.2) we get

$$
L=-\int_{0}^{\infty} e^{-u} z^{\prime}(u) d u+\int_{0}^{\infty}(2 u) e^{-2 u} d u+2 \int_{0}^{\infty} u e^{-2 u} d u \int_{0}^{u} e^{t} z^{\prime}(t) d t
$$


The value of the second integral is $\frac{1}{2}$. In the last integral we interchange the order of integration and perform the integration with respect to $u$. The result is

$$
\int_{0}^{\infty} e^{-t}\left(t+\frac{1}{4}\right) z^{\prime}(t) d t
$$

If we relabel the variable of integration in the last integral as $u$ and substitute into the defining relation for $L$ we get

$$
2 L=1+\int_{0}^{\infty} e^{-u} z^{\prime}(u) d u
$$

From this and from (10.6) we get $L=1-m$. Substituting this into (10.10) gives $K=5-2 \nu-9 m$. If we now use

$$
m=\left(1-\nu^{2}\right) / 2,
$$

which is a consequence of $(10.1)$, we find that $K=\left(9 v^{2}-4 v+1\right) / 2$ as asserted.

The next lemma characterizes the behavior of $z^{m}$ under the assumption that there is more than one quartic arch. Other results of this kind were given in $\S 9$ of [1]. The consequences of these results and the inequality (10.8) will lead to a contradiction.

LEMma 13. With the possible exception of the last component, $z^{m}$ changes sign in each component. In fact

$$
\text { signum } z^{m}\left(\alpha_{i}\right)=(-1)^{i}, \quad i=0,1,2, \ldots, N-1 .
$$

From (10.5) we get that $z^{m}(0) \geqq 0$ with equality holding if and only if $F(0)=0$. From (9.1) and Lemma 7 we see that $F(0)=0$ is impossible. Hence $z^{m}(0)>0$. If $z^{m}$ did not change sign in the interior of the first component $\left(0, \alpha_{1}\right)$, then since $z^{m}$ is linear we would have $z^{m}\left(\alpha_{1}\right) \geqq 0$. On the next component $\left(\alpha_{1}, \alpha_{2}\right), z(u)<0$, and so $z^{(\mathrm{iv})}(u)=I$. Hence $z^{m}(u)>0$ on $\left(\alpha_{1}, \alpha_{2}\right)$. Since $z^{\prime}\left(\alpha_{1}\right)<0$ it follows from Lemma 9 that $z^{\prime \prime}\left(\alpha_{1}+0\right) \geqq 0$. Since $z^{\prime \prime}(u)>0$ on $\left(\alpha_{1}, \alpha_{2}\right)$ we have $z^{\prime \prime}(u)>0$ on $\left(\alpha_{1}, \alpha_{2}\right)$. Hence $z^{\prime}$ is strictly increasing on $\left(\alpha_{1}, \alpha_{2}\right)$. Since $z\left(\alpha_{1}\right)=z\left(\alpha_{2}\right)$ and $z(u)<0$ on $\left(\alpha_{1}, \alpha_{2}\right), z^{\prime}$ must have a zero in the interior of $\left(\alpha_{1}, \alpha_{2}\right)$. Therefore, $z^{\prime}\left(\alpha_{2}\right)>0$ and there exists a third component $\left(\alpha_{2}, \alpha_{3}\right)$ on which $z(u)>0$. Hence by (5.5)

$$
z^{\prime \prime}\left(\alpha_{2}+0\right)=z^{\prime \prime}\left(\alpha_{2}-0\right)+2 I>0 .
$$

Since $z^{\prime}\left(\alpha_{2}\right)>0$, this contradicts Lemma 9. Therefore, $z^{m}\left(\alpha_{1}\right)<0$.

By repeating the argument we can next show that $z^{m}$ must change sign in the second component. We proceed inductively in this fashion up to the next to the last component. There we conclude by noting that if $z^{m}$ does not change sign we have $z^{\prime}\left(\alpha_{N}\right) \neq 0$, a contradiction.

11. Proof that $N=1$. In this section we shall show that the minimizing function has only one quartic arch. The formula for $q$ given in Theorem 3 then follows from Lemma 11. This leaves only the numerical determination of the constants to be found in $\$ 12$. 
Suppose that $N>1$. Then from Theorem 2 we have that $z(u)>0$ on the first component $\left(0, \alpha_{1}\right), z(u)<0$ on the second component $\left(\alpha_{1}, \alpha_{2}\right)$, and $z^{\prime}\left(\alpha_{1}\right)<0$. Since $z^{\prime}\left(\alpha_{1}\right)<0$ it follows from Lemma 9 that $z^{\prime \prime}\left(\alpha_{1}+0\right) \geqq 0$. From (5.5) we get

Hence

$$
z^{\prime \prime}\left(\alpha_{1}+0\right)=z^{\prime \prime}\left(\alpha_{1}-0\right)-2 I \text {. }
$$

$$
z^{\prime \prime}\left(\alpha_{1}-0\right)>2 I \text {. }
$$

From Lemma 13 it follows that there exists a point $\beta$ in $\left(0, \alpha_{1}\right)$ such that $z^{m}(\beta)=0$. Since $z^{\prime \prime}$ is a quadratic on $\left(0, \alpha_{1}\right)$ and $\left(z^{\prime \prime}\right)^{\prime \prime}=z^{1 \mathrm{v}}=-I$, it follows that $z^{\prime \prime}$ has a maximum at $\beta$. Since $z^{\prime \prime}(\beta)>z^{\prime \prime}\left(\alpha_{1}-0\right)$ it follows from (11.1) that

$$
z^{\prime \prime}(\beta)>2 I \text {. }
$$

On the interval $\left(0, \alpha_{1}\right)$ we have

$$
\begin{gathered}
z^{\prime \prime}(u)=-I u^{2} / 2+z^{m}(0) u+z^{\prime \prime}(0), \\
z^{m}(u)=-I u+z^{m}(0) .
\end{gathered}
$$

Since $z^{m}(\beta)=0$ it follows from (11.4) that $\beta=z^{m}(0) / I$. Substituting this value of $\beta$ into (11.3) and using (11.2) gives

$$
\left(z^{m}(0)\right)^{2} / 2 I>2 I-z^{\prime \prime}(0) .
$$

Using (10.3) and (10.4) we may rewrite this inequality in the following equivalent form:

$$
8 I^{2}+4 I\left(1-\nu^{2}\right)-(1-\nu)^{4}<0 .
$$

From (11.1) and from the fact that $z^{\prime \prime}$ is a quadratic on $(0, \alpha)$ with maximum at $\beta$ it follows that $z^{\prime \prime}(u)$ is positive on $\left(\beta, \alpha_{1}\right)$. Therefore $z^{\prime}$ is strictly increasing on $\left(\beta, \alpha_{1}\right)$. Since $z^{\prime}\left(\alpha_{1}\right)<0$ it follows that $z^{\prime}(\beta)<0$. If we substitute $u=\beta=z^{m}(0) / I$ into

$$
z^{\prime}(u)=-\frac{I u^{3}}{6}+\frac{z^{\prime \prime}(0)}{2} u^{2}+z^{\prime \prime}(0) u+z^{\prime}(0), \quad 0 \leqq u \leqq \alpha_{1},
$$

we get

$$
3 I^{2} z^{\prime}(\beta)=\left(z^{m}(0)\right)^{3}+3 I z^{\prime \prime}(0) z^{m}(0)+3 z^{\prime}(0) I^{2} .
$$

If we now use (10.2)-(10.4) we see that $z^{\prime}(\beta)<0$ implies

$$
12 I^{2}\left(1+\nu^{2}\right)-6 I(1-\nu)^{3}(1+\nu)+(1-\nu)^{6}<0 .
$$

We now multiply $(11.5)$ by $(1-\nu)^{2}$, add the resulting inequality to (11.6), and then divide by $I$. After some elementary simplifications we get

$$
I<(1+v)(1-v)^{3} / 2\left(5 v^{2}-4 v+5\right) .
$$

We now eliminate $I$ between this inequality and the inequality (10.8) to obtain, after simplification,

$$
24 v^{4}-31 v^{3}+33 v^{2}-9 v+1<0
$$


The left-hand side of (11.7) can be rewritten as follows:

$$
\left(1-\frac{9}{2} \nu\right)^{2}+\frac{51}{4} \nu^{2}\left(1-\frac{62}{51} \nu\right)^{2}+\frac{263}{51} \nu^{4}
$$

This expression is clearly nonnegative for all $\nu$. Hence (11.7) cannot be satisfied and the assumption that $N>1$ leads to a contradiction.

12. Numerical determination of constants. The quartic $q$ of Theorem 3 has the following properties:

$$
q(0)=0, \quad q^{\mathrm{iv}}(u)=-I, \quad q(\lambda)=q^{\prime}(\lambda)=0 .
$$

Hence $q$ has another positive root $r>\lambda$ and we can write

$$
q(u)=I u(\lambda-u)^{2}(r-u) / 24 .
$$

If we expand the right-hand side of (12.1) and compare coefficients of the powers of $u$ with those in the representation of $q$ given in Theorem 3, we obtain the equations

$$
\begin{aligned}
I \lambda^{2} r & =24 \nu, \\
I\left(\lambda^{2}+2 r \lambda+12\right) & =6\left(1-\nu^{2}\right), \\
I(r+2 \lambda) & =2(1-\nu)^{2} .
\end{aligned}
$$

Since $z(u)=0$ for $u \geqq \lambda$ and $q(u)>0$ on $(0, \lambda)$, it follows that $I=\int_{0}^{\lambda} q(u) d u$. Substituting (12.1) into the right-hand side of this equation and performing the integration gives

$$
24 / \lambda^{4}+\lambda / 30=r / 12 .
$$

Equations (12.2) and (12.3) were solved numerically to obtain the values of $I, \lambda, \nu$ given in Theorem 3. The value of $m$ is obtained from the value of $\nu$ by means of (10.1).

\section{REFERENCES}

1. L. D. Berkovitz and H. Pollard, A variational problem related to an optimal filter problem with self-correlated noise, Trans. Amer. Math. Soc. 142 (1969), 153-175.

PuRdue University,

LAFAYETTE, INDIANA 47907 